$\xi=1$ -

\title{
Fuzzy Logic Aware QoS Multicasting in MANETs with Load Balance
}

\author{
Nageswara Rao Sirisala ${ }^{1}$, Raswitha Bandi ${ }^{2}$ \\ ${ }^{1}$ Professor CSE Department, Vardhaman College of Engineering \\ ${ }^{2}$ MLR Institute of Technology,Hyderabad, Hyderabad,India \\ *Corresponding author E-mail: nagsirisala@gmail.com
}

\begin{abstract}
Mobile ad-hoc network (MANET) is a decentralized and infrastructure less network where a nodes can communicate with other nodes within the access region. Due to mobility node can enter and leave a network at any moment. Due to unstable nature of MANETs, the provision of Quality of Service (QoS) to the applications is a difficult task. In this paper, fuzzy logic enabled QoS multicast routing is proposed. Here energy, bandwidth and link expiry time are considered as a QoS parameters. The existing methods lost their performance in handling multi constrained QoS protocols, since defining the dynamic priorities among the multiple QoS parameters is not a trivial task. In the proposed method "Fuzzy Logic Aware QoS Multicasting in MANETs with Load Balance-FQML", this issue is overcome by using fuzzy logic. The Competency Factor of each intermediate node along the route is calculated by aggregating it's QoS parameters using fuzzy inference system. In the classical multicast protocols, some of the nodes in the multicast tree are overloaded by having multiple branches towards the destination nodes. This leads to partition of the multicast tree and degrades the performance of protocols. In the proposed method, this problem is handled by limiting the number of branches at intermediate nodes. The results are taken in network simulator-ns2, where the proposed method could measure less number of path failures and improved results than existing methods.
\end{abstract}

Keywords: Competency Factor; Fuzzy Logic; Multicast tree; Quality of Service

\section{Introduction}

MANETs(Mobile ad hoc networks) are the local area networks without certain infrastructure, where a node can do transactions and communication with all other nodes within it's radio coverage area. In MANET each node has freedom of moving from one location to another location, hence there is no fixed network topology. Networks are instantly can be formed and dissolved as per their needs. In the network, there is no centralised administration, each node can act as a router and takes its own decision. In MANET routing is the key feature, where the shortest route with qualified intermediate nodes has to be identified between source and destination for successful data transmission. By devising effective routing protocols, the performance of the applications can be increased.

In unicast routing a single source node sending the data to the single destination node, whereas in multicast routing (Group communication) a source node sending the data to the set of destination nodes in the same time intervals[10,11,12]. In multicast routing, multiple paths have to be identified from source to each destination node, i.e a multicast tree has to be formed by connecting source with all destination nodes. In a multicast tree, source sits in root position and all destination nodes are at leaf positions. Quality of Service (QoS) is the important issue to be addressed in MANET, where certain applications need minimum amount of resources like bandwidth and energy to get run successfully[4,5,6]. QoS multicast routing protocols works formation of multicast tree, where each possible path from source to each destination should satisfy all QoS requirements $[13,14,15]$. In multicast routing the intermediate nodes are forwarding multiple copies of source data to the set of destination, hence these nodes suffer lack of energy and bandwidth $[8,9]$. In this paper, the QoS parameters energy, bandwidth and link expiry time(LET) are considered in the multicast tree formation.

When a protocol handles multiple QoS parameters, the problem is defining the priorities among them. In this paper we used fuzzy logic to consider multiple QoS constrains based on network conditions. In defyzzification for each intermediate node, competency factor $(\mathrm{CF})$ is calculated. The proposed routing protocol establishes the path through the intermediate node with higher $\mathrm{CF}$ values. In multicast routing protocol, sometimes the multicast tree is formed with overloaded intermediate nodes with multiples branches towards destination. In that case, the resources of overburdened nodes are sonly drain out and it leads to partitions of multicast tree. This problem is addressed in the proposed method by limiting the number of branches in the tree (load balance).

\section{Related Work}

The multicast protocols can be classified as mesh based and tree based protocols [7]. In this section some of the QoS aware unicast(AQOR[19]) and multicast(MAODV[18],PMRP[16]) routing protocols are discussed. 


\subsection{Multicast Ad Hoc On-Demand distance Vector Protocol (MAODV)}

MAODV is the extention of unicast routing protocol that is AODV. Whenever a node wants to become a new member of multicast group, it sends the join RREQ to the neighbour nodes. If the nodes are group members then gives the reply (RREP) to the new node otherwise forwards the same to other nodes by adding its ID. Every group member receives join request and send the reply packets to the new receiver node. From received multiple RREP packets, new node selects the best group member in terms of hop count and joins the group through that node.

\subsection{Ad Hoc QoS On-demand Routing (AQOR)}

Ad Hoc QoS On-demand Routing follows the basic working principle of Adhoc Ondemand Distance Vector(AODV) protocol, with extension of QoS parameters. Here, whenever a source node wants to establish a path to destination, sends the Route Request(RREQ) packets to all of its neighbour nodes with delay and bandwidth needs. All the intermediate nodes verify their remaining resources, if they have sufficient amount of resources then they accept and forward the RREQ packet to next hop neighbours otherwise ignore them. Eventually, the destination node receives the multiple RREQ packets through multiple paths. Through the RREQ packets, a destination node can filter the unworthy routes. The destination node finally gives the route reply(RREP) to the request packet which has come across through most qualified path in terms of bandwidth and delay.

\subsection{Power Aware Multicast Routing Protocol (PMRP)}

PMRP is the extention of MAODV routing protocol, PMRP works based on two metrics $P_{\text {prediction }}$ and $P_{\text {remain }}$. $P_{\text {prediction }}$ is the estimation of required node power to forward source data $P_{\text {remain }}$ is the current remaining node power. In route discovery process, every intermediate node forwards the RREQ packet only if it is having enough power to handle the source data i.e $P_{\text {prediction }}<P_{\text {remain }}$.

\section{QoS Aware Multicasting with Topology Control and Load Balance (FQML)}

Here, section 3.1 describes the motivation/need of load balance in the multicast tree by avoiding formation of overladed intermediate nodes with multiple branches, which is not addressed in the existing methods. Section 3.2 describes the calculation of link expirer time between two nodes, calculation of node's power consumption in data forwarding and node's bandwidth with multiple branches in multicast tree. In section 3.3 fuzzy sets for the parameters LET, energy and bandwidth are discussed with the help of diagrams. Calculation of competency factor (CF) for each intermediate node using fuzzy logic is explained. In section 3.4, the proposed multicast routing algorithm is presented.

\subsection{Evaluation of QoS parameters}

In this section the calculation of QoS parameters like LET, Bandwidth and packet forwarding energy is discussed.

\subsubsection{Estimation of link Expiration Time(LET)}

The link expiry time between two nodes in the MANET depends on their present locations, velocities and their moving directions[16]. Let's consider two nodes are presently positioned at $\left(l_{1}, m_{1}\right)$ and $\left(l_{2}, m_{2}\right)$ and moving with speeds of $s_{1}$ and $s_{2}$ towards the directions $d_{1}$ and $d_{2}$. Two nodes are within d distance coverage area. Then their contact time is evaluated as in eq(1).

$$
L E T=\frac{\sqrt{d^{2}\left(g^{2}+i^{2}\right)-(g j-i h)^{2}}-(g h+i j)}{\left(g^{2}+i^{2}\right)}
$$

Where $g=s_{1} \cos d_{1}-s_{2} \cos d_{2}, h=l_{1}-l_{2}$,

$$
i=s_{1} \sin d_{1}-s_{2} \sin d_{2} \text { and } j=m_{1}-m_{2}
$$

\subsubsection{Energy Calculation}

A node forwarding energy is the sum of data receiving energy and forwarding energy[16]. Let's consider $E_{t r}$ and $E_{a}$ are the energies required for a node to activate transmitter/receiver and amplifier respectively then the forwarding energy in transmission of $\mathrm{m}$ bit data over the distance $r$ can be calculated as in eq (2)

$$
E_{\text {total }}(k)=E_{a} \times m \times r^{2}+2 \times\left(E_{t r} \times m\right)
$$

\subsubsection{Bandwidth calculation using TDMA}

According to TDMA[20], the available bandwidth at a node is sharing with the neighbour nodes with whom it is communicating. A $n_{i}$ can do the data transmission with the node $n_{j}$ in the time interval of $s_{t}$, if this time interval is not assigned either at $n_{i}$ or $n_{j}$ and not scheduled at any neighbour node of $n_{i}\left(n_{k}\right)$ for receiving data. It can be formulated as shown in the eq(3). Likewise the receiving slots of $n_{i}$ can be formulated as in eq(4).

$T S_{i}=\left\{s_{t} \in S: s_{t} \notin R S_{i}, s_{t} \notin T S_{i}, s_{t} \notin \bigcup_{n k \in N B_{i}} R S_{k}\right\}$

$R S_{i}=\left\{s_{t} \in S: s_{t} \notin R S_{i}, s_{t} \notin T S_{i}, s_{t} \notin \bigcup_{n k \in N B_{i}} T S_{k}\right\}$

\subsection{Estimation of Competency Factor(CF) using fuzzy logic}

An intermediate node Competency Factor is calculated using fuzzy logic where its QoS parameters LET, energy and Bandwidth are used as fuzzy input variables.

\subsubsection{Fuzzy logic system}

Fuzzy logic is good inference mechanism where the decisions can be taken when the available information is not precise[1,2,3,17]. Where the multiple input factors are aggregated in the phase of fuzzyfication and the final crisp output value is evaluated in the defuzzyfication phase. Both the phases work based on fuzzy rule base. This process is described in the figure1.

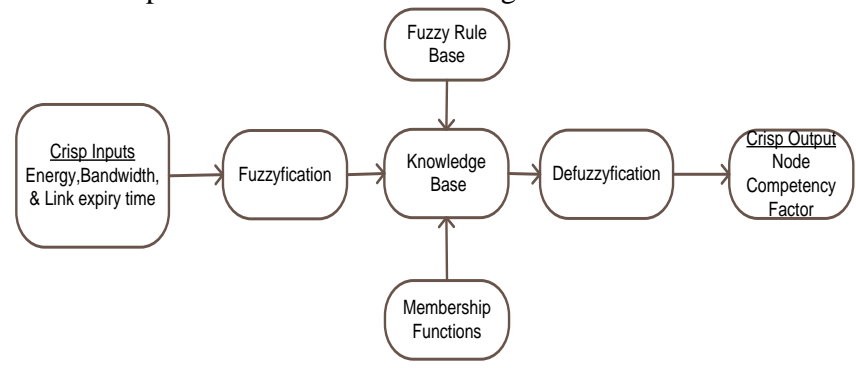

Figure 1: Fuzzy Logic in FQML 


\subsubsection{Fuzzification}

In fuzzyfication the input crisp values are converted into fuzzy set linguistic terms. Here each of input parameters (LET, energy and bandwidth) are divided into four fuzzy sets like very low, low, medium and high.

\subsubsection{Fuzzy membership functions}

Here the triangular membership functions are used since the attenuation rate of QoS parameters is linear. In Figure 2, the membership functions are shown for input(energy, Bandwidth and LET) and output parameters. Based on the simulation experiment setup the intervals of fuzzy set are considered as energy (0-20-4060Jouls), Bandwidth(0-1-2-3 Mbps), LET(0-30-60-90 Sec) and $\mathrm{CF}(0-0.330-0.66-1)$.



(a) Energy membership function

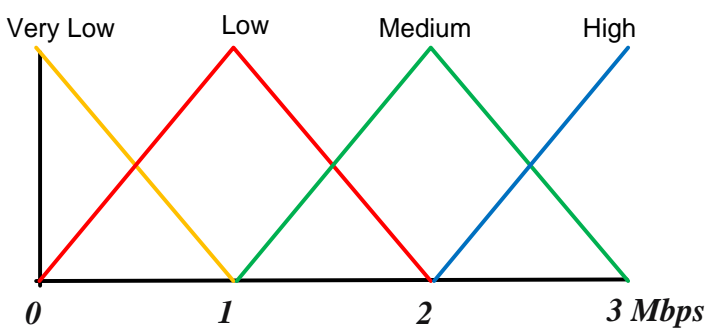

(b) Bandwidth membership function



(c) LET membership function

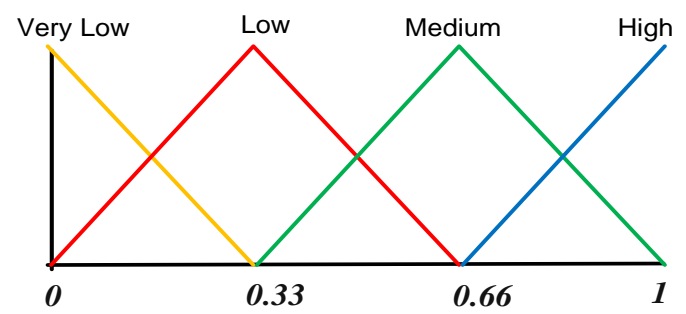

(d) CF membership function

Figure2: Triangular membership functions of fuzzy input variables

\subsubsection{Rule Base}

The fuzy rule base are framed to reflect the network conditions. Usually fuzzy rules follow the IF-THEN' format. In the below table some of the fuzzy rules are listed out with the combination of input and output variable.

TABLE 1: Fuzzy Rule Base

\begin{tabular}{|l|l|l|l|}
\hline Energy & Bandwidth & LET & CF \\
\hline Medium & Medium & Low & Low \\
\hline Very Low & Very Low & Very Low & Very Low \\
\hline Low & Low & Medium & Low \\
\hline High & Medium & Medium & Medium \\
\hline Medium & Medium & Low & Medium \\
\hline
\end{tabular}

Equation (5) is applied over the CF fuzzy set in the figure 4 and the finalised CF output value is 0,58 .

3.3 Uneven load distribution in the classical muticast tree construction

Lets consider the multicast tree is formed using classical musticast routing method as shown in Fig 5. Where node $S$ is a source and E,F,C are set of destination nodes. Here the node B is overloaded 
as it is the common intermediate node along the paths to all destination nodes from source node. Hence the resources of node B drain out very soon and it becomes dead node. In that case the multicast tree has to be reconstructed to find alternative paths to all destination nodes. Hence there is a necessity of avoiding overloaded intermediate nodes to achieve load balance in the multicast tree. The proposed algorithm can address this problem by limiting the number of branches in the multicast tree.

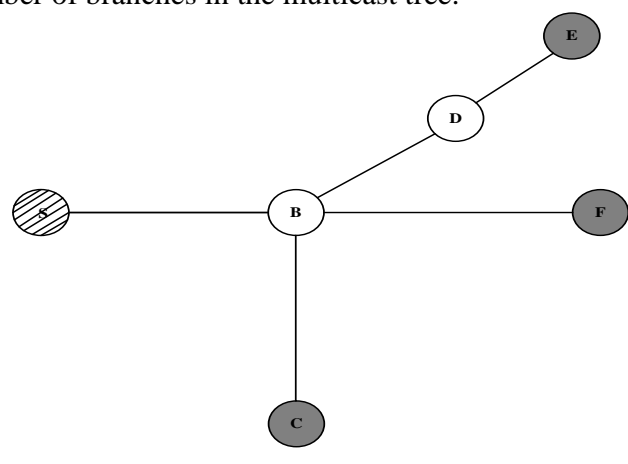

Figure 5: multicast tree

\subsection{FQML muticast routing protocol.}

After computation of QoS parameters and node competency factor, the FQML protocol is run to find the paths to set of destinations with higher $\mathrm{CF}$ values.

\subsubsection{Route Discovery Process}

Step 1: source node, first sends the RREQ packets to all of its 1hop nodes with required level of QoS values. After receiving RREQ packets, an intermediate node verifies its available resources. If it has sufficient amount of resources as per source requirement then computes its $\mathrm{CF}$ value and adds it to the request packet before forwarding. Otherwise simply ignores the RREQ packet saying it is not capable of handling request.

Step 2: all the RREQ packets collect the intermediate node CF values in their journey to destination nodes.

Step 3: all the destination nodes wait till all the RREQ packets are reached. From multiple RREQ pakets, destination node selects and gives the reply(Route Reply-RREP) to the packet which has come across through the path with higher $\mathrm{CF}$ values. Each destination maintains the alternative path information and sets the $\mathrm{AP}($ Alternative) field to 1 , if alternative path is available. (the format of RREP packet is like in [18] with extra field AP).

Step 4: while an intermediate node receiving multiple RREP packets from different destinations (i,e going to be overloaded). It verifies its available resources. If it is not capable to handle data transmissions to the multiple destinations, then it drops the RREP packets with AP field was set to 1.

Step 5: after the RREP is rejected, an intermediate node sends the route reply failure (RREP_F) packet to the concerned destination node. the destination node selects the alternative path.

Step 6: eventually the multicast tree is formed from source node to all destination nodes without having overloaded intermediate nodes; hence the network life time will be extended

\subsection{An example of multicast routing in FQML}

In Fig 6, the multicast routing is explained over the example network topology. Where S is the source node and the E,F,C are the destination nodes. In Fig 6(a), node S sends the RREQ packets to all the destinations but all the destination nodes selects the paths through node B and sends the RREQ packets. In Figure 6(b), node $B$ instead of accepting all RREP packets(i.e overloaded) it rejects the RREP packet from node $\mathrm{E}$ and sends back route failure packet
RREP_F. On receiving failure packet, node E resends the RREP packet to the source through A. In Fig 6(c), the load balanced multicast tree is formed.

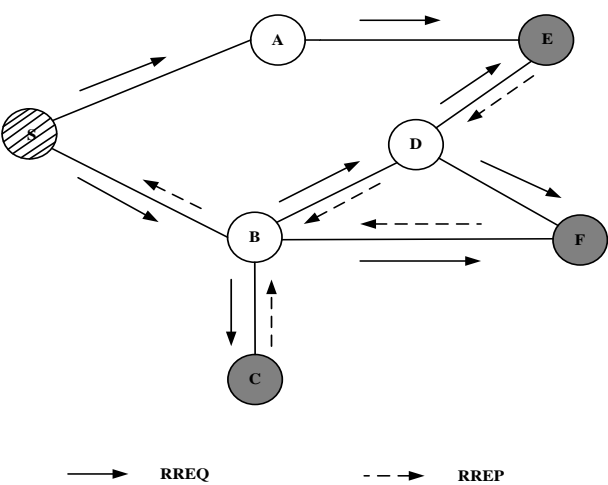

Figure 6: (a): Route Request in FQML.

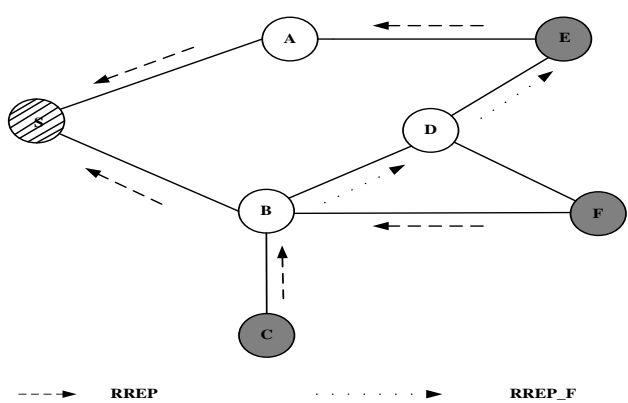

Figure 6: (b): Route Replay Phase in FQML

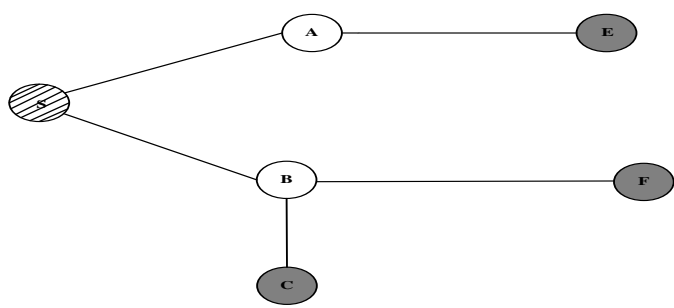

Figure 6: (c) Multicast Tree in FQML.

\section{Simulation Results}

The simulation is conducted in ns 2.34 network simulator, where the performance of the proposed method FQML is verified over the existing methods MAODV and PMRP. The experiments are conducted in the area of $1400 \times 1400$ over the period of $600 \mathrm{sec}$. Here the results are taken for the number of nodes varying from 10-60 nodes and the node velocities are considered from 0 to 40 $\mathrm{m} / \mathrm{sec}$. Node initial energy is $60 \mathrm{~J}$ and transmission range is $250 \mathrm{~m}$.

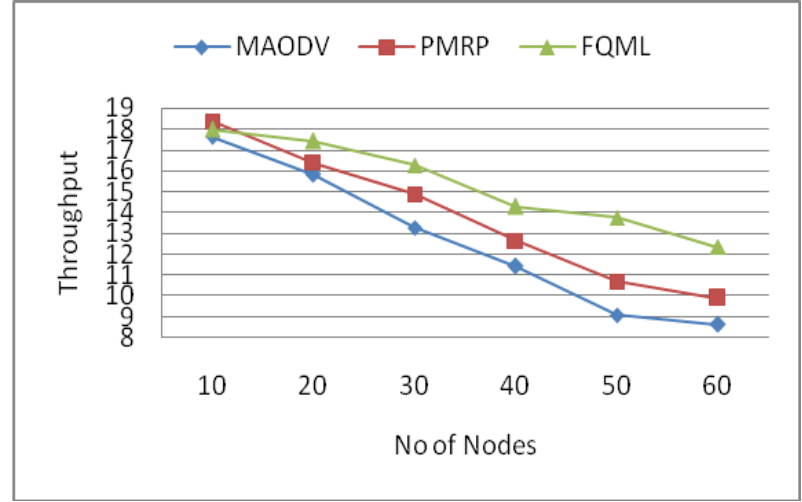

Figure 7: Number of nodes Vs throughout 
In figure7, when the number of nodes are increased the bandwidth at the intermediate nodes are reduced and hence the throughput is reduced. But in the proposed method the number of branches in the multicast tree is reduced hence the bandwidth is high and throughput is improved.

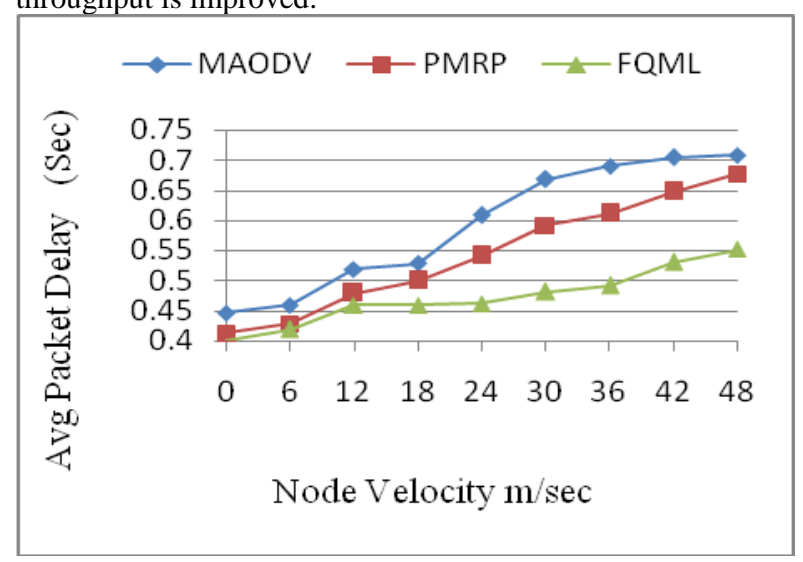

Figure 8: Node velocity Vs Average packet delay

In figure 8 , when node velocity is increased, some of the intermediate nodes move beyond the access region of the network hence established paths get disconnected and alternative paths are requied. It leads to delay in packet delivery time at destination. but the proposed method considers only stable nodes along the path, hence it can extends the network life time and can reduce the packet delivery time at destination.

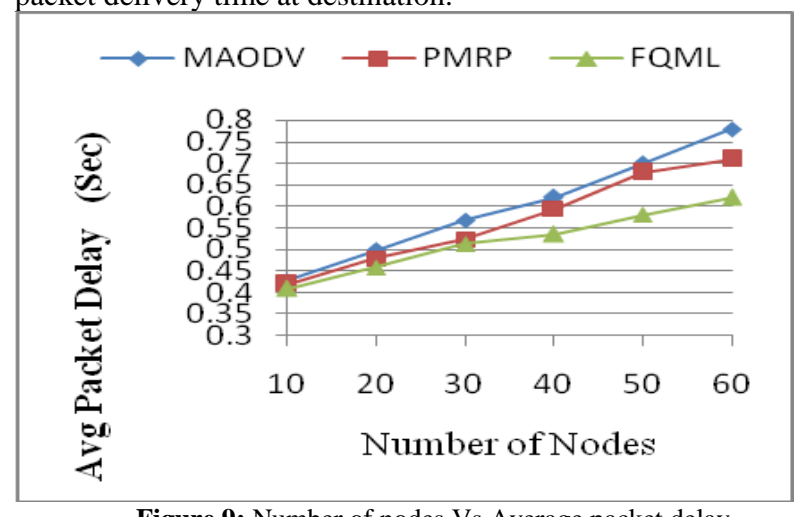

Figure 9: Number of nodes Vs Average packet delay

In figure 9: the average packet delay is getting increased while the network size is increased. When the number of nodes is high then network maintenance is high. In case of path failure, the routing protocol has to spend much time in establishing alternative path which delays packet delivery time at destination. But in the proposed method, each destination has the information of alternation path to source node, hence it does require much time in establishment of alternative path. So it can better performance than existing methods

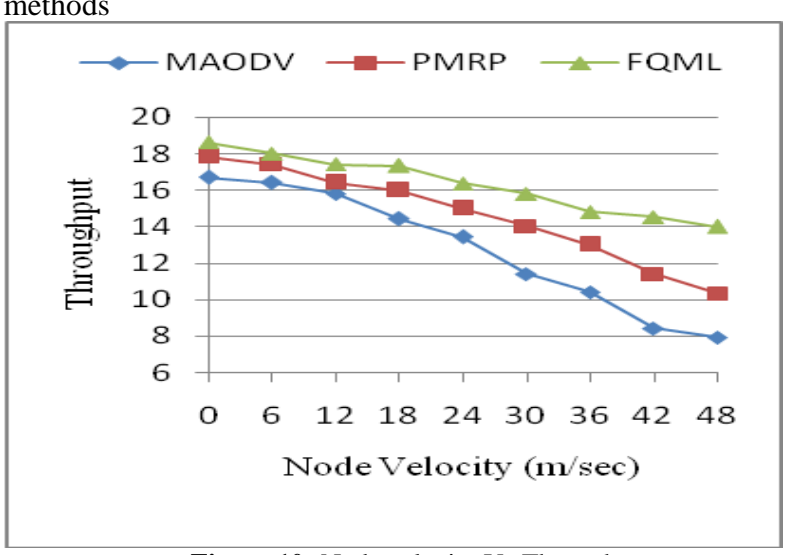

Figure 10: Node velocity Vs Throughput
The through is reduced when nodes are moving at higher velocities. At higher velocities of nodes, paths may get disconnected frequently. Which can effects the packet delivery ratio at destination. But the proposed method considers the stable nodes and has the quick mechanism of alternative path establishment, hence can give better results than existing methods.

\section{Conclusion}

The proposed method "Fuzzy Logic Aware QoS Multicasting in MANETs with Load Balance-FQML" is a multicast routing protocol, which considers energy, bandwidth and node mobility as QoS parameters. The fuzzy logic is used to compute the each node Competency Factor (CF) in the network by considering QoS parameters. FQML can establish the paths from source to multiple destinations through the intermediate nodes with higher $\mathrm{CF}$ values. Hence it can reduce the path breaks and can extend the network life time. The proposed method can avoid the overloaded intermediate nodes in the multicast tree formation; hence the load is evenly distributed in the network. In the simulation results the proposed method could measure the improved results over the existing methods.

\section{References}

[1] NageswaraRao Sirisala and C.Shoba Bindu. A Novel QoS Trust Computation in MANETs Using Fuzzy Petri Nets, International Journal of Intelligent Engineering and Systems, Vol.10, No.2, (2017), pp 116-125.

[2] NageswaraRao Sirisala and C.Shoba Bindu. Recommendations Based QoS Trust Aggregation and Routing in Mobile Adhoc Networks, International Journal of Communication Networks and Information Security (IJCNIS), Vol 8, No 3, (2016), pp 215-220.

[3] Fei Hao; Geyong Min; Man Lin; Changqing Luo; Yang, L.T. 2014, "MobiFuzzyTrust: An Efficient Fuzzy Trust Inference Mechanism in Mobile Social Networks", IEEE Transactions on Parallel and Distributed Systems, vol.25, no.11, pp.2944-2955.

[4] Zhexiong Wei; Tang, H.; Yu, F.R.; Maoyu Wang; Mason, P., 2014 "Security Enhancements for Mobile Ad Hoc Networks With Trus Management Using Uncertain Reasoning," IEEE Transactions on Vehicular Technology, vol.63, no.9, pp.4647-4658.

[5] Sirisala, NageswaraRao.; C.Shoba Bindu. 2014 "Weightage based trusted QoS protocol in Mobile Adhoc Networks", IEEE Global Conference on Wireless Computing and Networking (GCWCN), pp.283-287.

[6] Lei Ju, Edwin H. M. Sha, Hui Xia, Zhiping Jia, Xin Li, 2013 “Trust prediction and trust-based source routing in mobile ad hoc networks" Ad Hoc Networks Elsevier,Vol. 11, No. 7, pp. 2096-2114.

[7] NageswaraRao Sirisala and C.Shoba Bindu. Uncertain Rule Based Fuzzy Logic QoS Trust Model in MANETs, International Conference on Advanced Computing and Communications -ADCOM, (IITM PhD forum), (2015), pp.55-60.

[8] Ruifeng Zhang, Olivier Berder, Jean-Marie Gorce, Olivier Sentieys 2012 "Energy-delay trade off in wireless multihop networks with unreliable links",,Ad Hoc Networks v10, pp1306-1321.

[9] Nicola Costagliola - Pedro Garçia López · Francesco Oliviero · Simon Pietro Romano, 2012 "Energy- and Delay-Efficient Routing in Mobile Ad Hoc Networks". Mobile Network Applications v17 pp:281-297.

[10] Luo Junhai, Ye Danxia, Xue Liu, and Fan Mingyu, 2009 "A Survey of Multicast Routing Protocols for Mobile Ad-Hoc Networks". IEEE Communications Surveys \& Tutorials, (Volume:11, Issue: 1), Page(s):78 - 91 .

[11] Mina Masoudifar, 2009 “A review and performance comparison of QoS multicast routing protocols for MANETs" Ad Hoc Networks 7(6), pp:1150-1155.

[12] Ben-Jye Chang and Szu-Liang Kuo, 2009 "Markov Chain Trust Model for Trust-Value Analysis and Key Management in Distributed Multicast MANETs", IEEE Transactions on Vehicular Technology, vol.58, no.4, pp.1846-1863.

[13] Chia-Cheng Hu, Eric Hsiao-Kuang Wu, Gen-HueyChen, 2008 "bandwidth-Satisfied Multicast Trees in MANETs", IEEE Transactions On Mobile Computing, Vol. 7, No. 6,pp:712-723. 
[14] Guo, S. 2007 "Energy-aware multicasting in wireless ad hoc networks: A survey and discussion', Computer Communications", Volume 30 Issue 9, Pages 2129-2148.

[15] Huayi Wu, Xiaohua Jia, 2007 “QoS multicast routing by using multiple paths/trees in wireless ad hoc networks", Ad Hoc Networks Volume 5 Issue 5, pages 600-612.

[16] Nen-Chung Wang, Yung-Fa Huang · Yu-Li Su 2007 “A PowerAware Multicast Routing Protocol for Mobile AdHoc Networks With Mobility Predictio", Wireless Personal Communications, Vol 43:pp.1479-1497.

[17] Jing Nie, Jiangchua Wen, Ji Luo, Xin He, Zheng Zhou 2006 “An adaptive fuzzy logic based secure routing protocol in mobile ad hoc networks", Fuzzy Sets and Systems, Volume 157, Issue 12, 16 Pages 1704-1712.

[18] Baolin, S. and Layuan, L. 2005 "On the reliability of MAODV in ad hoc networks", IEEE international symposium on microwave, antenna, propagation and EMC technologies for wireless communications, Vol. 2, pp. 1514-1517.

[19] Q. Xue and A. Ganz, "Ad Hoc QoS On-demand Routing (AQOR) in Mobile Ad hoc Networks," J. Parallel and Distrib. Comp., vol. 62, no. 2, Feb. 2003, pp. 154-65.

[20] Kuei-Ping Shih. 2002 "A TDMA-based bandwidth reservation protocol for QoS routing in a wireless mobile ad hoc Network", IEEE International Conference on Communications Conference Proceedings ICC (Cat No 02CH37333) ICC-02. 